\title{
Trismus due to bilateral mandibular coronoid hyperplasia.
}

\author{
AJ Gibbons, AJ Byrne, SJ Key
}

\begin{abstract}
Bilateral mandibular coronoid hyperplasia is a rare cause of restricted mouth opening. Diagnosis of the condition prior to general anaesthetic is essential, as oral intubation may be impossible. The reported case illustrates the role of computed tomography in assessment of the disorder and effective treatment by coronoidectomy.
\end{abstract}

\section{Introduction}

Bilateral mandibular coronoid hyperplasia is a condition of insidious onset characterised by a painless limitation of mouth opening. The enlarged coronoid processes impinge on the zygomatic arches causing a physical obstruction to mandibular movements. Diagnosis of the condition is confirmed by an orthopantomograph (OPG). Computed tomography further details the region's anatomy and may reveal additional zygomatic pathology.

The following case report demonstrates the dramatic improvement in mouth opening which can be achieved through surgical intervention.

\section{Case Report}

A 28 year old male Sergeant of European descent was referred to the Department of Oral and Maxillofacial Surgery, Princess Alexandra's Royal Air Force Hospital, Wroughton, for assessment of his restricted mouth opening. The patient first noticed the problem at 16 years of age and had
Wg Cdr A J Gibbons MA (CANTAB), FDSRCS, FRCS, RAF Specialist Registrar Oral \& Maxilofacial Surgery

Dr A J Byrne

MRCP, FRCS

Consultant Anaesthetist

Mr S J Key

FDSRCPS, FRCS

Staff Grade Oral \&

Maxillofacial Surgery

Morriston Hospital, Swansea, S. Wales, SA6 6NL

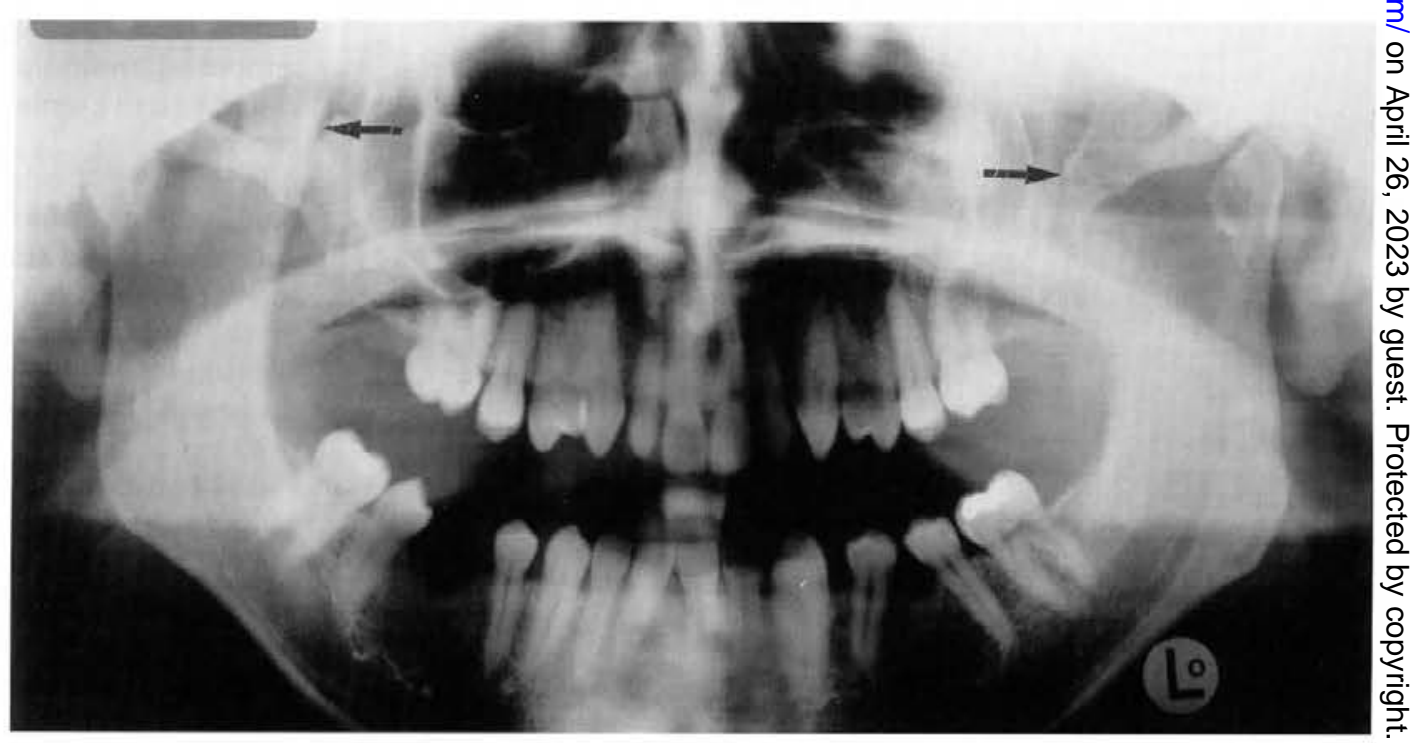

Fig 1. Panoramic radiograph showing coronoid process (arrowed). previously been unsuccessfully treated at a $\frac{\overline{\frac{}{O}}}{\bar{\omega}}$ dental hospital with bite raising and $\frac{\vec{\Phi}}{\varrho}$ interdental screw appliances.

On clinical examination the patient's inter- incisal opening was limited to $15 \mathrm{~mm}$ and the $\overrightarrow{0}$ top of the right coronoid process could be $\vec{\omega}$ palpated above the zygomatic arch. An OPG $\stackrel{\text { ? }}{\text { ? }}$ (Figure 1) showed unequal enlargement of the coronoid processes. Three-dimensional computed tomography (3-D CT) images $\vec{A}$ were taken to detail more clearly the morphology and relationships of the coronoid processes (Figure 2). These $\vec{\overrightarrow{ }}$ revealed a large extosis of the right zygomatic arch at the point of contact with the coronoid $\vec{\overrightarrow{ }}$ process (Figure 3).

Coronoidectomies were subsequently performed under general anaesthetic using a fibreoptic technique to establish ${ }^{\circ}$ endotracheal intubation. In view of the gross.enlargement of the coronoid process and additional zygomatic pathology, an external temporal/pre-auricular approach was made to the right side. The zygomatic arch was sectioned, pedicled from the masseter and $\varrho$ following coronoidectomy, reconstituted $\overrightarrow{\overrightarrow{0}}$ with mini-plates. Opening improved to $\frac{3}{5}$ $30 \mathrm{~mm}$ allowing an intra-oral approach to coronoidectomy on the left side.

Post-operatively the patient could open to $40 \mathrm{~mm}$ and was encouraged to use an interdental screw appliance to help prevent $\frac{\widehat{D}}{\partial}$ fibrosis. Histological examination of the $\stackrel{\varrho}{\rightleftharpoons}$ excised coronoid revealed normal mature bone, consistent with a diagnosis of $\underline{3}$. hyperplasia. 


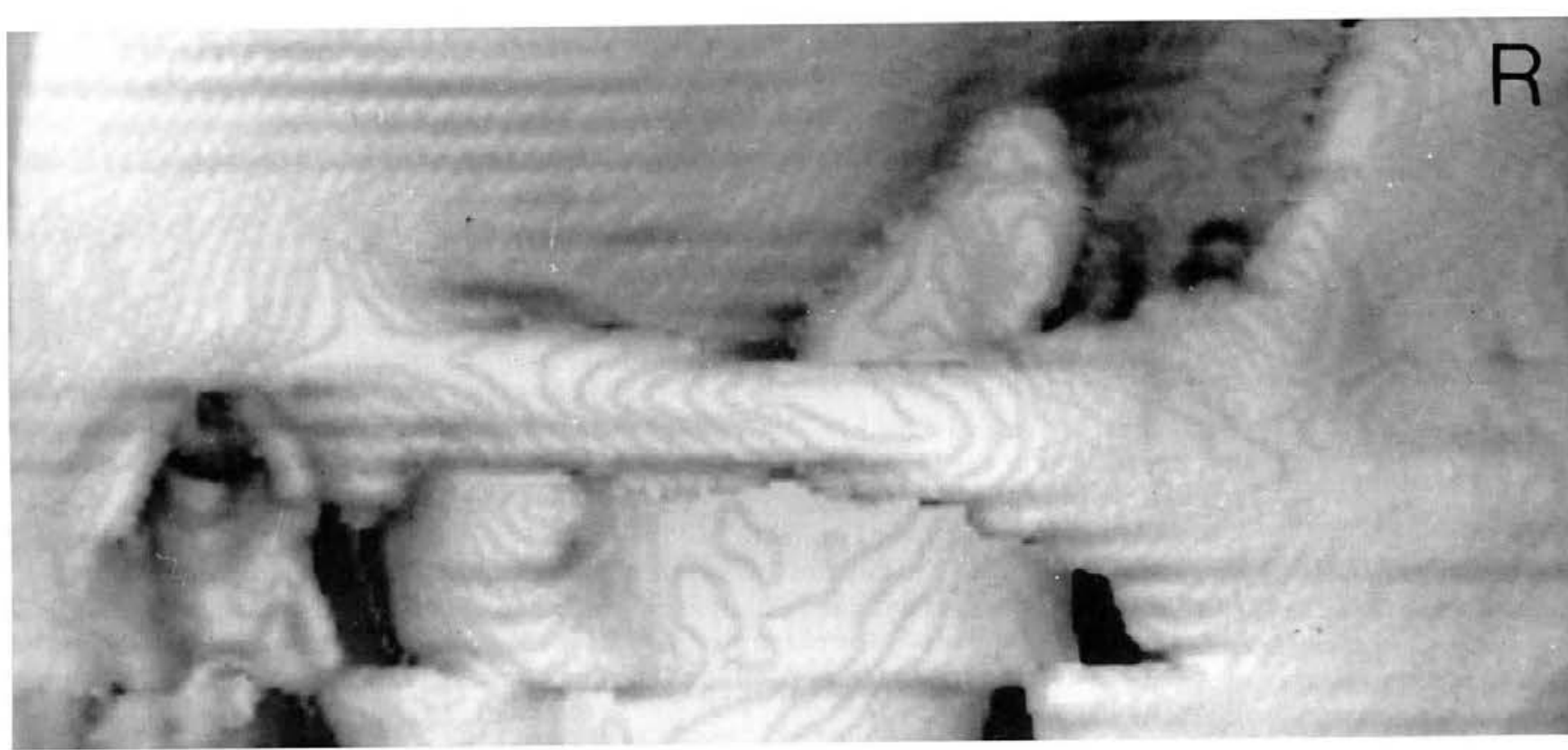

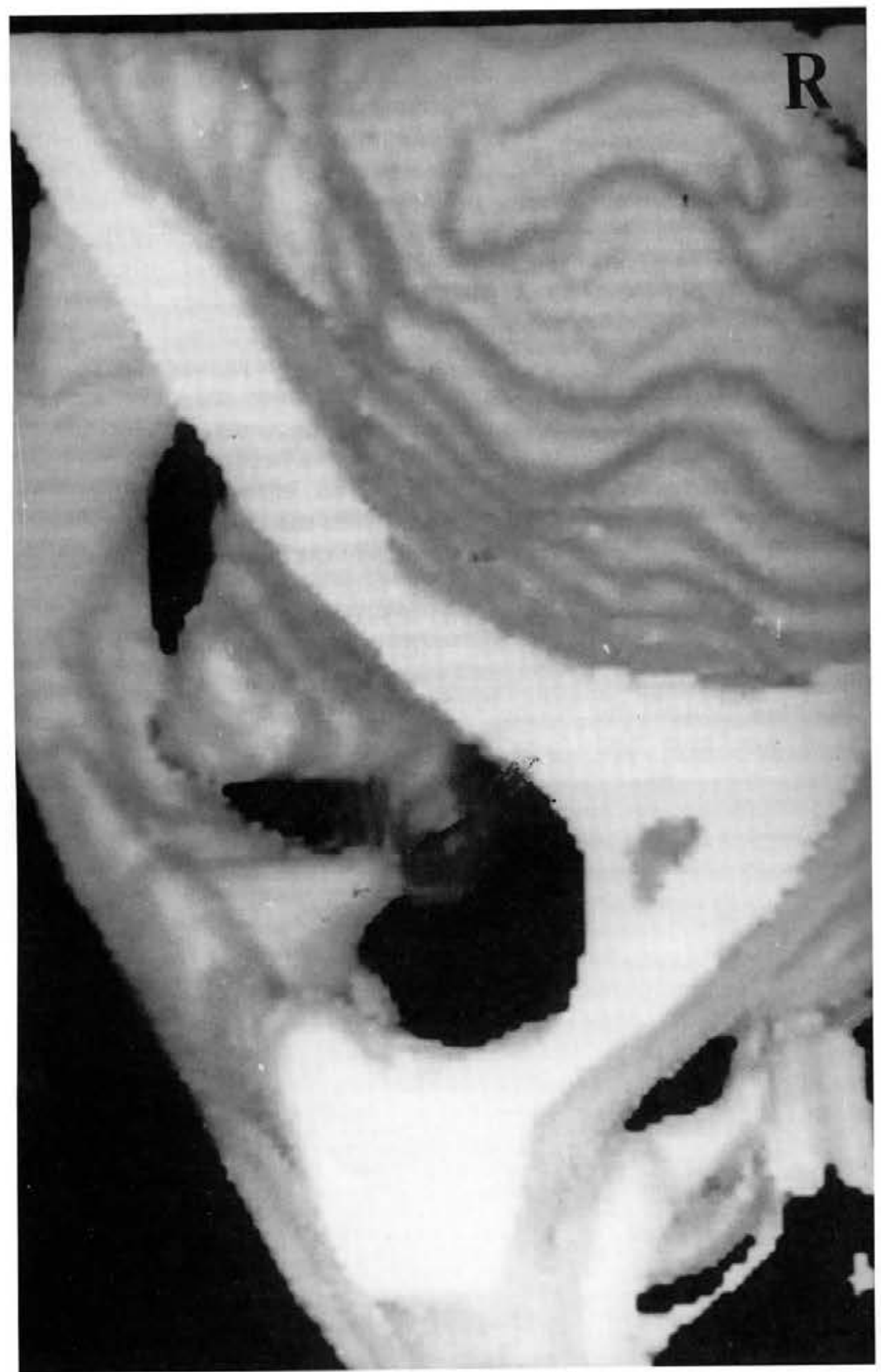

Fig 3. 3-D CT scan showing right zygomatic arch extosis (arrowed).
The patient's improved opening haso remained stable and he has no evidence of $\underset{-}{\supset}$ regrowth of the coronoid processes.

\section{Discussion}

Bilateral mandibular coronoid is insidious in onset and not associated with pain or deformity. Therefore, patients may be unaware that they have limited moutho opening (1). The condition may be life threatening. Rusconi and Brusatti (2) ing 1963 reported a patient involved in a road产 traffic accident where tracheal intubation $=$ proved impossible and an emergency tracheostomy was required. Subsequently a diagnosis of bilateral mandibular coronoidơ hyperplasia was established as the cause of his restricted mouth opening.

The aetiology of bilateral mandibular coronoid hyperplasia remains unclear. The condition is more common in males, with an onset in puberty indicating a possiblé endocrine influence (1). In animal models hyperactivity of the temporalis muscleo triggered by hypomobility of the temporomandibular joint has been found to옹 cause reactive enlargement of the coronoid processes (3).

Previously bilateral and unilatera mandibular coronoid hyperplasia have been defined as two distinct entities (1). However many cases display at least some abnormalityo of the opposite side and it is likely that continuous spectrum exists, from the pures unilateral case to complete involvement of both processes (4).

Bilateral mandibular coronoid hyperplasia may not be as rare as formerly believed. Of the 88 cases of the disorder in the Westerno literature 23 were identified at one unit alone (4). In a review of 163 patients with limited opening of the mandible, Isberg et al (5) found that $5 \%$ cases were due to् enlargement of the coronoid process. As the 
condition is neither painful nor associated with facial deformity, patients often fail to seek advice. Clinicians unfamiliar with the disorder may also fail to consider the diagnosis.

The Levandoski pantographic analysis of OPGs may be used to determine if a coronoid processes is enlarged (6). When the ratio of the length of the coronoid process to the condylar process on the radiograph is greater than 1.1 a diagnosis of coronoid process hyperplasia is suggested and further investigation of the condition is warranted.

Computed tomography accurately demonstrates the detailed anatomy of the region including the precise extent of the enlarged coronoid processes and their relationships to the zygomatic arches. This information assists the surgeon in evaluating whether an external temporal/pre-auricular or intra-oral approach to coronoidectomy is required.

Surgery is the only successful form of treatment. Awake fibreoptic intubation is the safest anaesthetic technique to use on patients diagnosed with this condition. In cases of extreme trismus, an external temporal/pre-auricular approach allows better surgical access to the region and avoids restrictive intra-oral scarring.

Regrowth of the coronoid processes following intra-oral coronoidectomies may occur. This may be due to regeneration of bone with haemotomas (7) or the influence of abnormal temporalis muscle fibres (4). Consequently, long term review of treated patients is recommended.
When assessing the airway prior to surgeryo if there is restricted mouth opening mandibular coronoid hyperpalsia althoug廷 rare, should be considered in the differentiat diagnosis.

\section{Acknowledgments}

We thank Air Commodore T W Negus? Consultant in Oral Maxillofacial Surgery fox permission to present his patient, Aiq Commodore J Reid, Director of Denti Services (RAF) for his consent to publis this paper.

\section{References}

1. Rowe NL. Bilateral development hyperplasia of the mandibular coronoid process. Br f Oral Surg 196 $\overrightarrow{\text { W }}$ 1: $90-104$.

2. Rusconi L, Bruscatti R. Restricted opening of the mouth from symmetrical bilateral hyperplasia of th coronoid processes. F Oral Surg 1974; 32: 452-456

3. Isberg AM, McNamara Jr JA, Carlson DS, Isacsso走 G. Coronoid process elongation in rhesus monkeyळ (Macaca mulatta) after experimentally induced mandibular hypomobility. Oral Surg Oral Med Oraz Pathol 1990; 70: 704-710.

4. McLoughlin PM, Hopper C, Bowley $\mathrm{NB}^{\text {? }}$ Hyperplasia of the mandibular coronoid process: $\mathrm{AD}$ analysis of 31 cases and a review of the literature. Oral Maxillofac Surg 1995; 53: 250-255.

5. Isberg A, Isacsson G, Nah KS. Mandibulaథ coronoid process locking. A prospective study of frequency and association with interna derangement of the temporomandibular joint. Orat Surg Oral Med Oral Pathol 1987; 63: 275-279.

6. KubotaY, Takenoshita Y, Takamori K, Kanamoto M Shirsuna K. Levandoski pantographic analysis ip the diagnosis of hyperplasia of the coronoid proces: Br 7 Oral Maxillofac Surg 1999; 37: 409-411.

7. Smyth A G, Wake MJC. Recurent bilateral coronoi\& hyperplasia: an unusual case. $\mathrm{Br} \mathcal{F}$ Oral Maxillofa $\overrightarrow{\overrightarrow{0}}$ Surg 1994; 32: 100-104. 